\title{
On the nature of the transition region from the chromosphere to the corona of the Sun
}

\begin{abstract}
H. Peter*
Kiepenheuer-Institut für Sonnenphysik, Schöneckstrasse 6, 79104 Freiburg, Germany

Received 5 December 2000 / Accepted 10 May 2001

Abstract. One of the keys to understand coronal heating is to understand the (magnetic) structure of the atmosphere below the respective coronal features. The EUV emission lines formed in the thin transition region from the chromosphere to the corona present a sensitive tool to study the structure and dynamics of the lowermost corona and its connection to the chromosphere. Data from the SUMER spectrograph on SOHO show for the first time that broad components are a common feature of emission line profiles formed from some $40000 \mathrm{~K}$ to $10^{6} \mathrm{~K}$. The contribution of that tail component to the total intensity of the line exhibits a trend with line formation temperature that peaks in the middle transition region with smaller contribution at high and low temperatures. The line width of the tail component shows a monotonic increase with temperature that is consistent with a passing Alfvén wave, which is in contrast to the trend in width of the line core. Together with previous observations this presents evidence that the line core and the tail component are formed in radically different physically regimes. It is proposed that these are small closed loops and coronal funnels, respectively, with the latter being the footpoints of large coronal loops. The new results on the structuring of the transition region will improve understanding on heating the corona and accelerating the solar wind.
\end{abstract}

Key words. Sun: transition region - Sun: corona - stars: coronae - line: profiles

\section{Introduction}

In a "classical" spherical-layer picture the transition region between the chromosphere and the hot corona is extremely thin (below $0.01 \%$ of the Sun's diameter). Despite that this region is of great diagnostic value to study the coronal heating problem: it forms the lower boundary of the corona, and all the energy that is ultimately heating the corona and accelerating the solar wind has to cross this thin layer. Therefore the structure and dynamics of the transition region contains information also on the physics of the corona. Revealing the (magnetic) structure of the transition region is thus one of the major keys to the understanding of coronal heating. This paper presents new data analysis techniques applied to observations with the Solar and Heliospheric Observatory (SOHO) showing details of a multi-component transition region with radically different heating of the various structures. This provides important clues and raises new questions on the coronal heating problem.

The most prominent features of the (solar) chromosphere are bright structures forming the chromospheric network and outlining the boundaries of the supergranular flow cells (Fig.1). The magnetic field in the lower chromosphere is concentrated in this network. As

\footnotetext{
* e-mail: peter@kis.uni-freiburg.de
}

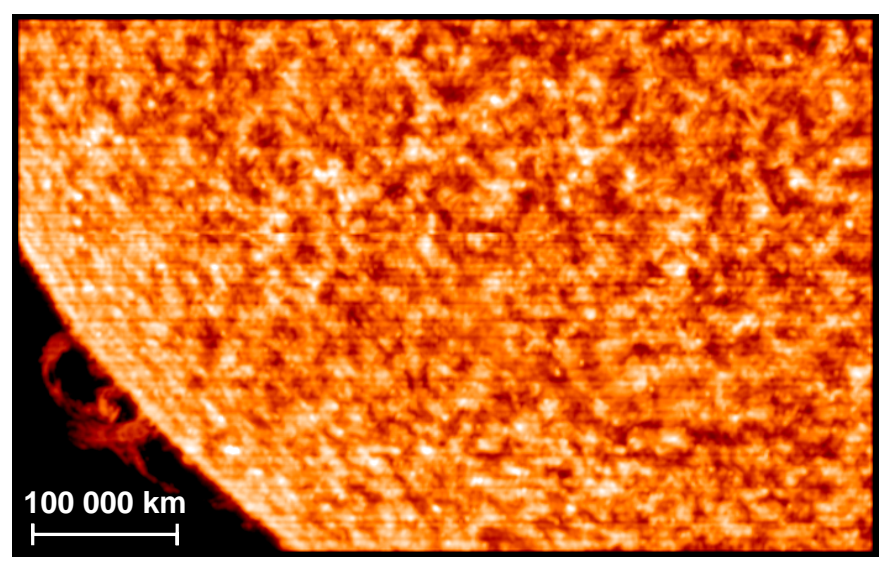

Fig. 1. The solar transition region at $60000 \mathrm{~K}$ as seen in the C III (977 $\AA$ ) emission line. South-eastern part of a SUMER full-disk scan from 28 January 1996. The patchy pattern is the chromospheric network with a typical cell size of $20000 \mathrm{~km}$. A prominence can be seen above the limb.

the corona is filled with magnetic field this implies that only a small fraction of the solar surface, about $1 \%$, is magnetically connected to the corona. This led Gabriel (1976) to propose a transition region confined to funnels formed by the magnetic field, with a rapid expansion at about $3000 \mathrm{~km}$ above the photosphere. This morphology 
implies that the network structure, clearly visible at low temperatures, vanishes towards higher temperatures, as was observed, e.g., with Skylab (Reeves 1976). Later investigations showed that smaller transition region structures also exist below the funnels (Dowdy et al. 1986) and that transition region emission is enhanced above small bipoles indicating the existence of small loops (Porter et al. 1987).

It is important to distinguish between the contribution of transition region emission from small-scale structures like small loops, which are not magnetically connected to the "global" corona, and emission from larger structures like funnels that can be viewed as the base of the corona, either of large coronal loops forming the "quiescent corona" (Priest et al. 1998) or of magnetically open structures in coronal holes. This two- or multi-component structure confronts us with a severe line-of-sight problem as the larger funnels span over the smaller network loops.

To deconvolve the small- and large-scale structures images providing only the line intensity information, i.e. filtergrams, are not particularly useful, because, e.g., they do not allow one to distinguish between photons coming from different altitudes in the atmosphere. But with the aid of spectroscopy one is in principle able to deconvolve emission from optically thin regions, if either the (Doppler) line shifts or widths of the respective (basically Gaussian) line profiles are significantly different. As an example, differences in line shift have been used to identify explosive events (Dere et al. 1989; Innes et al. 1997).

Already data from the EUV spectrograph on Skylab in the mid-seventies showed that besides a narrow Gaussian component ("line core") the profiles of C III, Si IV and C IV emission lines formed in the middle transition region contain a broad second Gaussian component (Kjeldseth Moe \& Nicolas 1977). In the following the latter one will be called tail component as it is of importance only in the tail of the line profiles (cf. Fig. 2). The tail components were estimated to be a factor of about 3 to 4 broader than the line core components contributing about $30 \%$ to the total line emission. Almost 20 years later data from the High Resolution Telescope and Spectrograph (HRTS) allowed speculation on the broad components being a signature of unresolved explosive events (Dere \& Mason 1993). Stellar observations revealed that broad components in C IV and Si IV are also common among solar-like stars and that the contribution of the broad component increases with stellar activity (Wood et al. 1997). However, the stellar data have no spatial resolution and the Skylab data very poor spatial resolution $(1500 \times 45000 \mathrm{~km}$ on the Sun), while the HRTS data mentioned above were acquired during a rocket flight, allowing only rather short observing sequences.

This situation changed with the Solar and Heliospheric Observatory (SOHO), including the EUV spectrometer Solar Ultraviolet Measurements of Emitted Radiation, SUMER (Wilhelm et al. 1995). The instrument provides the possibility to acquire large raster scans with spectral resolution and sufficient signal-to-noise ratio allowing one to perform reliable double Gaussian fits of the spectral profiles. So far this has been done systematically only for the C IV (1548 $\AA$ ) line by Peter (2000).

In that paper a large raster scan $\left(540^{\prime \prime} \times 300^{\prime \prime}\right)$ of the mid-latitude quiet Sun was analysed. The data provide the full spectral profiles of C IV (1548 $\AA$ ). A single and a double Gaussian fit were applied to all spectra in the data set. The comparison of the single and double Gaussian fits by using $\chi^{2}$ showed that a single Gaussian fit is sufficient in the inter-network regions. In contrast a double Gaussian fit is needed in the bright network structures, with a line core component and a tail component being about a factor of 3 broader and a factor of 3 fainter than the core component. The tail component shows large clumps of blueshifted emission, which is also in contrast to the line core. Scatter plots of line shift and width vs. intensity differ significantly for the core and tail components (in the network), and are again different from the single Gaussian line profile parameters in the inter-network.

This observation led Peter (2000) to the conclusion that the source regions of the spectral components are heated by different means. One has to distinguish at least three physical components of the transition region. (1) The core component originates from small loops within the network; (2) the tail component originates from coronal funnels that emerge from in-between the small network loops and are footpoint regions of large coronal loops spanning across one or more network cells; (3) the single Gaussian inter-network emission is produced in regions where upward propagating acoustic shocks hit the canopy formed by the expanding coronal funnels.

The aim of the present paper is to extend these findings to other transition region emission lines, leading to a temperature dependence of the line fit parameters for the core and tail component. This will allow further insight into the processes actually leading to the heating of the various structures. In the following Sect. 2 the observations are presented together with a description of the data reduction. In Sect. 3 the observational results will be discussed. In Sect. 4 an attempt will be made towards an interpretation of the findings with regard to the heating and structure of the transition region. Section 5 will conclude the paper.

In this paper the following notation will be used. The observed line profiles in the network have a double Gaussian profile, with a narrow core, called core component (CC), and a wider component named tail component (TC). A single Gaussian fit will be abbreviated by SG. The radiance, i.e. intensity integrated over wavelength $\lambda$ measured in $\left[\mathrm{W} \mathrm{m}^{-2} \mathrm{sr}^{-1}\right]$, in the core component will be denoted $I_{\mathrm{CC}}$ and the one in the tail component by $I_{\mathrm{TC}}$. The total radiance from the line is given by $I_{\text {tot }}=I_{\mathrm{CC}}+I_{\mathrm{TC}}$. The strength or the contribution of the tail component to the total radiance will be denoted by $R=I_{\mathrm{TC}} / I_{\mathrm{tot}}$. The line width, $w$, will be given in Doppler widths of the Gaussian components, $\exp \left(-\lambda^{2} / w^{2}\right)$. It is related to the full width at half maximum by $F W H M=2 \sqrt{\ln 2} w$. The line width $w$ will be given in a velocity scale. Please note that this does not imply that the line width is mainly 


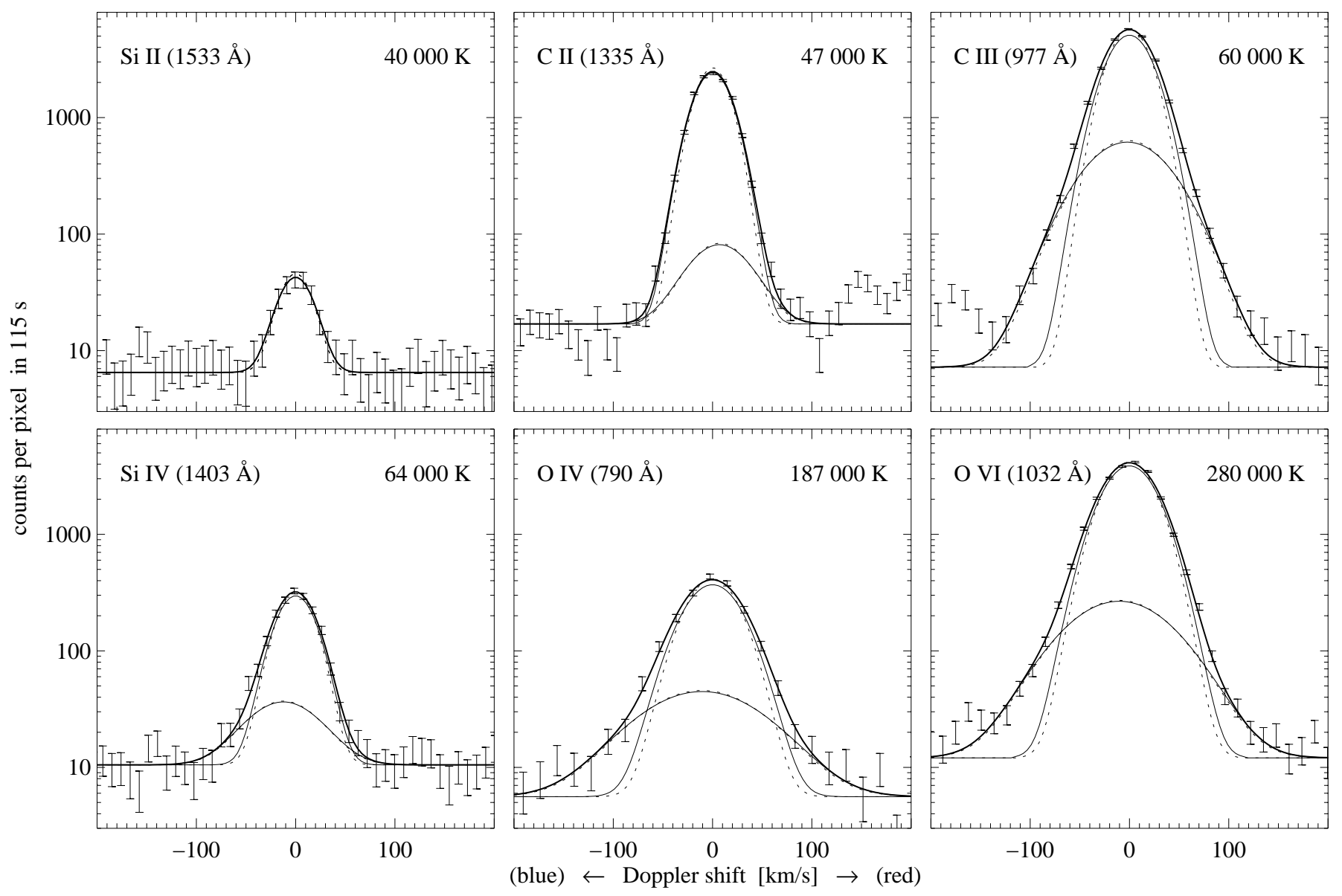

Fig. 2. Sample spectral profiles of transition region lines in the network. The lines and their formation temperatures are given with the plots. Each spectrum represents one spatial pixel of the SUMER instrument $\left(1^{\prime \prime} \times 1^{\prime \prime} \approx 750^{2} \mathrm{~km}^{2}\right.$ on the Sun). The observed spectra (uncertainties following Poisson statistics) are shown as bars. The thick lines represent double Gaussian fits, the thin lines the narrow core and the wide tail component as resulting from the fit. The thin dotted lines show the two components after subtraction of the instrumental broadening. The wavelength is given in a Doppler shift scale with the position of the line core set to zero shift. See Peter \& Judge (1999) for absolute Doppler shifts.
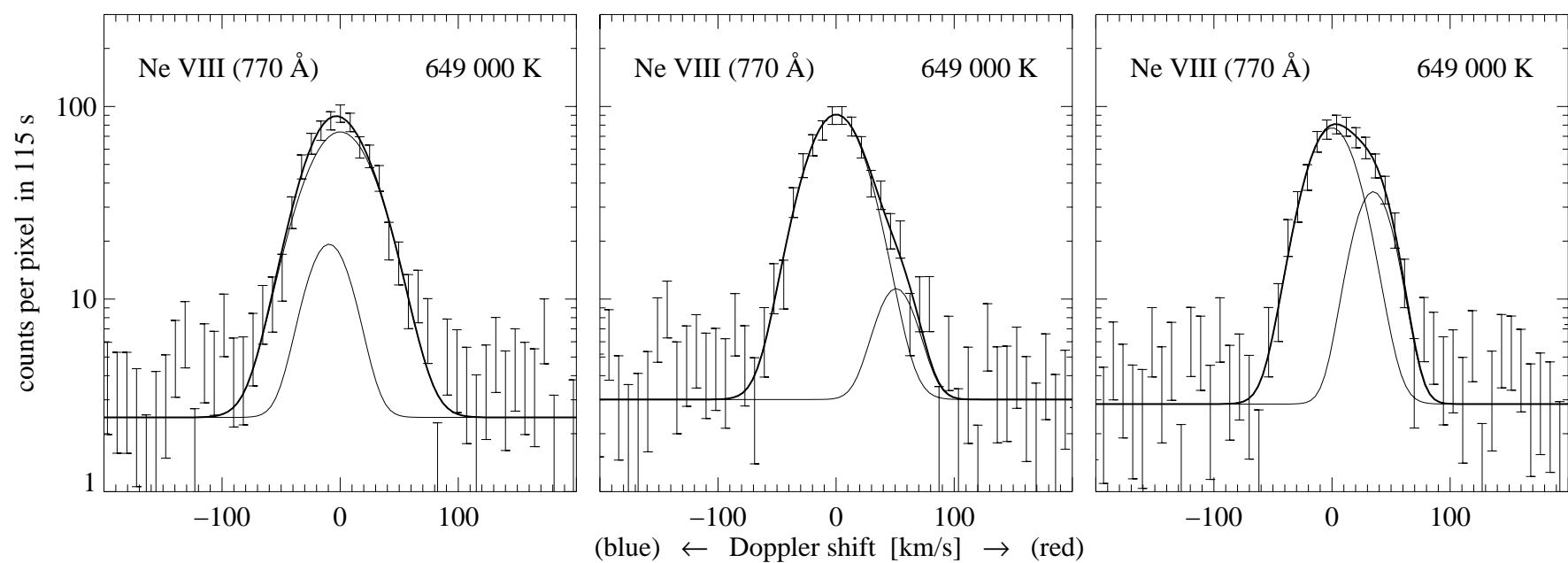

Fig. 3. Same as Fig. 2, but for three sample spectra of Ne VIII $(770 \AA)$ in the network. While the left example shows a second component of comparable line position and width compared to the line core, the other two examples show sort of a "bump" in the red wing of the line profile. In contrast to the profiles of the other lines shown in Fig. 2 this feature is quite typical for Ne VIII. 

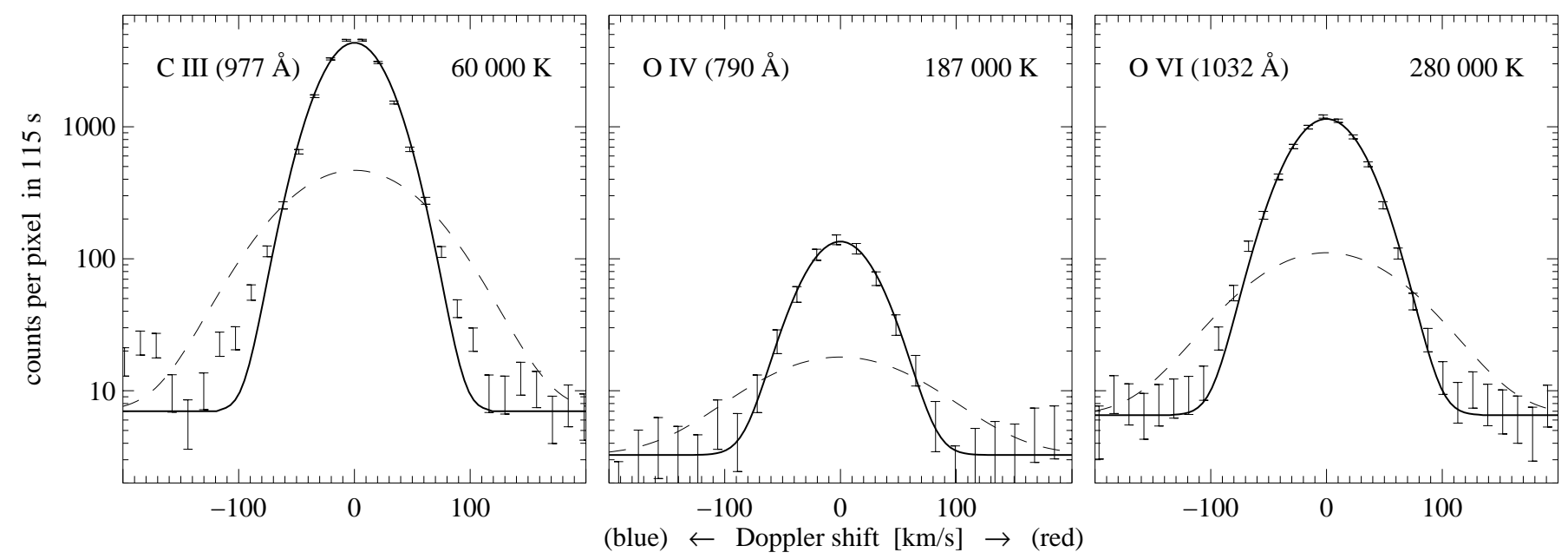

Fig. 4. Sample spectral profiles of transition region lines in the inter-network. Same as Fig. 2, but only for sample spectra of C III, O IV and O VI in the non-magnetic inter-network. Here single Gaussian fits are sufficient, even for the lines that show very clear tail components in the network as displayed in Fig. 2. The dashed lines show Gaussian profiles as they would be expected, if a tail component of comparable strength as for the network would be present also in the inter-network. In these inter-network spectra such tail components are not present (note the logarithmic intensity scale).

due to mass motions, but could also be due to thermal or wave motions. The line position will be given in a velocity scale, too, and will be denoted by $v$. All line shifts will be given relative to the respective core component, i.e. $v_{\mathrm{CC}}=0$.

\section{Data analysis}

\subsection{Observations}

As outlined in the introduction spectra with rather high signal-to-noise ratio are needed to observe a weak tail component. In the case of $\mathrm{C}$ IV the tail component is up to three times broader and three times fainter than the core component, i.e. the peak intensity of the tail component is only $1 / 10$ of the peak of the core component. If the tail component is centred on the core component a deviation from a single Gaussian shape, i.e. the tail component, is detectable only at least three Gaussian widths of the core component $w_{\mathrm{CC}}$ away from line centre, i.e. about $w_{\mathrm{TC}}$ off line centre (compare also the sample profiles in Fig. 2). There the intensity of the tail component dropped down to $\sim \exp (-1)$, i.e. to about $1 / 3$ of the peak of the tail component. In the case of $\mathrm{CIV}$ this is about $1 / 30$ of the peak intensity of the line profile. The conclusion is that for $\mathrm{C}$ IV a signal-to-noise ratio of 30 or better is needed to be able to detect the tail component. For the $\mathrm{C}$ IV line on the SUMER detector this implies that the exposure time is about $100 \mathrm{~s}$ or longer, resulting in count rates of 200 to 300 counts per pixel at line centre and less than 10 counts per pixel in the continuum for network locations.

In general one should have a signal-to-noise ratio of 100 or better to detect a tail component. The above discussion implies also that the line peak-to-continuum ratio should be above 100, too, to detect a tail component. This specification significantly narrows down the pool of emission lines that are suitable for the present study. Only in strong clear lines is enough information contained in the wings to apply a reliable double Gaussian fit. Finally less than a dozen lines in the SUMER spectral range $(500 \AA-$ $1600 \AA$ ) fulfil this requirement. Many other strong lines that could have ensured a better temperature coverage turn out to have weak blends in the wings or lines close by, which prevent a reliable fit of a tail component.

One of the major advantages of the data set used by Peter (2000) in the previous study on C IV was the huge amount of data allowing for statistically significant results. However, those big raster scans of SUMER with long exposure times take a long time to acquire - for the C IV data set more than 8 hours. Consequently only a very few of those large raster scans exist. To achieve good temperature coverage one therefore has to use other data sets.

For the present analysis a so called "ref-spec" was used. Such a data set consists of many individual exposures covering the full spectral range of SUMER. The spectra were taken at disk centre with the $1^{\prime \prime} \times 300^{\prime \prime}$ SUMER slit on 12 August 1996, i.e. during solar minimum, with an exposure time of $115 \mathrm{~s}$. The observed quiet Sun region covered bright network as well as inter-network structures. In Figs. 2 and 3 sample spectra from network regions are shown in the lines used for the present study. For the analysis of the C IV line the results of Peter (2000) were used and re-calculated where necessary.

Just like C IV all lines in the 50000 to $300000 \mathrm{~K}$ temperature range show a tail component in the network. The samples shown in Fig. 2 are typical for all network locations. For the inter-network regions the line profiles do not show any indication of a tail component with a strength comparable to the network regions (cf. Fig. 4). The Ne VIII line at $770 \AA$ in the network does not show a "regular" tail component, but rather "bumps", preferentially in the red wing (Fig. 3). These "pathological" profiles will be further discussed in Sect. 3.3. 


\subsection{Data reduction}

The original data are corrected for flatfield and geometric distortion of the detector image by standard SUMER IDL procedures. A detailed discussion on the limits of the wavelength calibration due to the geometric distortion can be found in Peter \& Judge (1999). The line widths were corrected for instrumental broadening (about $50 \AA$ corresponding to $15 \mathrm{~km} \mathrm{~s}^{-1}$ at $1000 \AA$ ) by using the SUMER IDL software con_width_funct_2. In Fig. 2 the core and the tail components corrected for instrumental broadening are over-plotted as dotted lines.

In the present paper no attempt was made to apply an absolute wavelength calibration, but all line shifts are given relative to the shift of the core component. This is motivated by the conclusion of Peter (1999) and Peter \& Judge (1999) that a reliable statistically significant "mean" absolute Doppler shift of the lines can be obtained only if a large enough data set is analysed. Otherwise the "solar noise", i.e. variations from one location on the Sun to the other, will lead to a distorted view. In addition, the main emphasis of the present paper is on the width of the lines and not their shift. The absolute line shifts of the line profile as a whole, which is essentially the same as the shift of the core component (cf. Sect.2.5, Table 1), are thoroughly studied by Peter \& Judge (1999).

The aim of the data analysis is to deconvolve a line profile consisting of a strong narrow Gaussian and a faint wide Gaussian component. As discussed in Sect. 2.1 one expects to need a signal-to-noise (or line-to-continuum) ratio of 30 to 100 to detect the tail component. As can be seen from the sample spectra in Fig. 2 to 4 even the strongest line on the SUMER detector, C III (977 $\AA)$, has a signal-to-noise ratio of well below 1000 at the exposure times used here, and the ratio is much worse for the other lines. Thus one needs a very robust mechanism to extract both the line core and the tail component.

To perform the Gaussian fits a Genetic Algorithm based optimisation was used (Charbonneau 1995), which proved very robust with similar tasks (Peter 1999, 2000). While a "classical" optimisation like the SIMPLEX algorithm is a local optimisation and needs a quite good initial guess to find the "true" optimal solution, a global algorithm like the Genetic Algorithm proves to be much more robust in finding the optimal solution even in noisy data. Therefore it is the ideal tool for the present task of fitting the bright core component and the faint wide tail component simultaneously. The output of the Genetic Algorithm is used as input for a classical local algorithm (IDL curvefit), to achieve faster convergence and to get a (local) estimate of the error of the fitted parameters. Both algorithms are based on minimising the reduced $\chi_{\mathrm{r}}^{2}$ (Bevington \& Robinson 1992). Using this method a single and a double Gaussian fit was applied for each individual spectral profile.

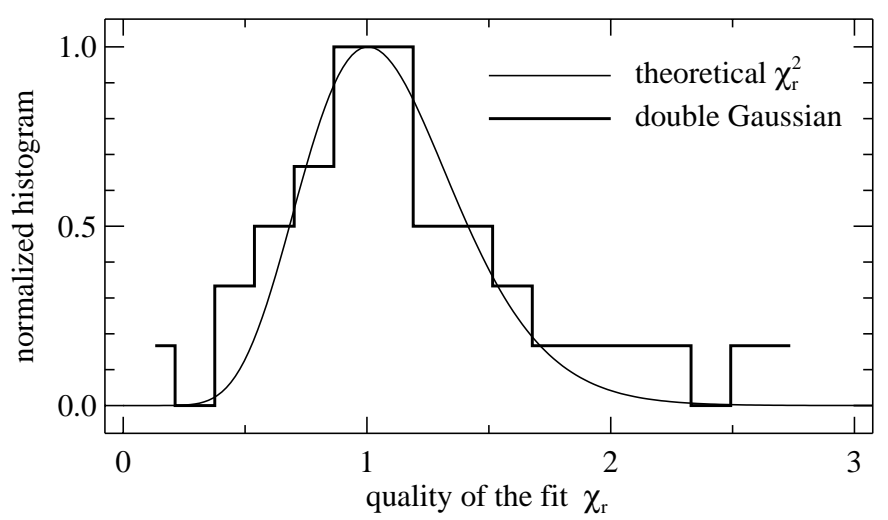

Fig. 5. Distribution of the reduced $\chi_{\mathrm{r}}^{2}$ for all double Gaussian fits in the network for C III, Si IV, O IV, and O VI (thick line). The thin line shows the theoretical optimal distribution.

\subsection{Single and double Gaussian fits}

For an optimisation problem the $\chi^{2}$ test is a reliable tool to check the quality of the fit. The reduced $\chi_{\mathrm{r}}^{2}$ is defined as

$\chi_{\mathrm{r}}^{2}=\frac{1}{[N-n]} \sum_{i=1}^{N} \frac{\left(d_{i}-m_{i}\right)^{2}}{\sigma_{i}^{2}}$,

where $d_{i}$ and $\sigma_{i}$ are the data and the respective errors at $N$ data points, and $m_{i}$ are the values of the fit at these points, with the fit having $n$ parameters. Here the $d_{i}$ are the intensities at $N$ wavelength pixels around the line, the errors $\sigma_{i}$ are given by Poisson statistics, and the model $m_{i}$ is a single or double Gaussian fit with a continuum, i.e. $n=4$ or $n=7$. The fit has $[N-n]$ degrees of freedom.

For a large number of optimisations (here: spectra) the reduced $\chi_{\mathrm{r}}^{2}$ are distributed as

$$
P_{\mathrm{r}}=\left(\chi_{\mathrm{r}}^{2}[N-n]\right)^{([N-n] / 2-1)} \frac{\exp \left(-\chi_{\mathrm{r}}^{2}[N-n]\right)}{2^{[N-n] / 2} \Gamma([N-n] / 2)}
$$

(see e.g. Bevington \& Robinson 1992). In Fig. 5 this theoretical optimal distribution is plotted as a thin line for the present problem of a double Gaussian fit $(n=7)$, for $N=40$ spectral pixels. The median value of the distribution is about $\left\langle\chi_{\mathrm{r}}^{2}\right\rangle \approx 1$ and the standard deviation of the $\chi_{\mathrm{r}}^{2}$ is about $\operatorname{stddev}\left(\chi_{\mathrm{r}}^{2}\right) \approx 0.5$. To check the quality of the fits a good criterion is to calculate the median value and the standard deviation of $\chi_{\mathrm{r}}^{2}$ for the double and single Gaussian fits of each of the lines and compare them to the theoretical optimal values of 1 and 0.5 . These values are listed in the rightmost column of Table 1 , which are for the spectra from network locations.

Inspection of the resulting $\chi_{\mathrm{r}}^{2}$ shows that in the network locations for all the lines, except for the cold Si II, Si IV, and the hot Ne VIII line, the double Gaussian fit is significantly better than the single Gaussian fit, and the double Gaussian fit is close to the theoretical optimal values (median and standard deviation). To emphasise the quality of the double Gaussian fits in the network the distribution of $\chi_{\mathrm{r}}^{2}$ for the lines of C III, Si IV, O IV and O VI is shown in Fig. 5 as a thick line. It is very close to the theoretical optimal curve (thin line) as given in Eq. (2). 
In the case of the Si II line, which is the coldest line in the sample, the algorithm is not able to fit a broad tail component at all. This is a clear indication that this line does not show a tail component down to the detection limit of the current method.

For the coronal Ne VIII line a second spectral component is found in the network, but it is a "bump", mostly in the red wing of the profile, with a width comparable to or smaller than the width of the line core. The second Gaussian component fitted to the Ne viII profiles is not a wide tail component as for the other transition region lines. The behaviour of Ne VIII will be discussed separately in Sect. 3.3.

\subsection{Possible influence of line blends}

It can almost be ruled out that the broad tail components are due to line blends, i.e. other weaker lines in the close vicinity of the respective line, which "mimic" a tail component. As all the "regular" lines from the chromosphere and transition region in the SUMER spectral range have line widths below about $25 \mathrm{~km} \mathrm{~s}^{-1}$, one blending line would not be sufficient to explain the broad tail components with widths of $50-100 \mathrm{~km} \mathrm{~s}^{-1}$.

Instead one would need at least two blending lines offset about $50 \mathrm{~km} \mathrm{~s}^{-1}$ towards the red and blue with respect to the line of interest. However, for two reasons there is no indication that this is the case. First, an inspection of line lists does not show blending lines with sufficient strength at the desired wavelengths. And second, a triple Gaussian fit with two narrow blends in the wings generally is less significant than a double Gaussian fit with a broad tail component.

The situation might be different for the coronal Ne viII line, where there is no broad tail component, but a "bump" in the line profile. A closer inspection shows that it is also questionable if this is due to a blend (Sect. 3.3).

\subsection{Line fit parameters}

The parameters of the single and double Gaussian fits are listed in Table 1 . There the median values of the respective parameter for all line profile fits in the network of the respective line are given along with the error of the fit and the standard deviation. The standard deviation is basically the "solar noise", i.e. variations in the observed region from one location to the other.

In almost all cases the error of the fit is smaller than the "solar noise". The bars in the plots of the fit parameters in Figs. 6 to 10 show the combined uncertainty of the error of the fit and the "solar noise", the length of the bars basically reflects the solar variability in space.

A comparison of the fit parameters for the single Gaussian (rows labeled SG) and the core component of the double Gaussian fit (CC) in Table 1 shows that the single Gaussian fit is a good representation of the line core component. This is not surprising, as the optimisation al- gorithm is minimising $\chi_{\mathrm{r}}^{2}$ as defined in Eq. (1), putting a higher weight at the high intensity values, i.e. at the line core. Thus the results for the line core allow a comparison to earlier studies performing only single Gaussian fits or a momentum analysis of the line profile.

\section{Observational results}

\subsection{Multi-component transition region spectra}

The present data show for the first time that a wide tail component is a general feature of all transition region emission lines studied and not only of those formed at about $10^{5} \mathrm{~K}$, like $\mathrm{C}$ IV and Si IV. This proves also that the tail components are not simply a feature of Li-like ions, i.e. they are not simply an artefact of atomic physics. These data allow one to investigate the line width and the contribution of the tail component to the total emission of the line as a function of temperature throughout the transition region. This provides new constraints on the structure and the heating of the transition region and corona.

Confirming the result of a previous study by Peter (2000) that the tail component of CIV is not (or only rarely) present in the inter-network, the present study extends this conclusion to the other transition region lines. For this reason all the following results apply only to quiet Sun network conditions.

\subsection{Variation of line fit parameters with temperature}

Contribution of the tail component. The strength of the tail component as a function of temperature is shown in Fig. 6. It is defined as $I_{\mathrm{TC}} / I_{\mathrm{tot}}$, where $I_{\mathrm{TC}}$ and $I_{\mathrm{tot}}$ are the intensity of the tail component alone and the total intensity of the line. Plotted are the mean values (in per cent) taken from spectra from the network. The bars basically show the standard deviation of the distribution of the derived parameters, i.e. the "solar noise" (cf. Sect. 2.5). While tail components are not present in the lowest transition region (below $30000 \mathrm{~K}$ ), their strength increases up to $\approx 30 \%$ in the middle transition region. Towards the corona the strength is decreasing.

Line width variation. The non-thermal line width $\xi$, also called non-thermal velocity, is defined through $w^{2}=$ $2 k_{\mathrm{B}} T / m+\xi^{2}$, where $w$ is the Gaussian width of the Gaussian on a velocity scale, and $k_{\mathrm{B}}, T$ and $m$ are Boltzmann's constant, temperature and atomic mass. For large line widths, like for the tail component, the $\xi$ are practically the same as the $w$. For a further discussion of the non-thermal line width see Sect.4.1.

The mean non-thermal line width $\xi$ as a function of temperature is shown in Fig. 7 for the core components (rectangles) and the tail components (bars). Again, the bars represent the "solar noise". The non-thermal width of the core component has a maximum in the middle transition region, and the present data compare well with 
Table 1. Results of the single and double Gaussian fits to the observed spectra in the network. Besides the ion and the wavelength the formation temperature of the line on a logarithmic scale, $\log T_{\mathrm{e}}[\mathrm{K}]$, and the ion sound speed at that temperature, $v_{s}=\left(k_{\mathrm{B}} T_{\mathrm{e}} / m\right)^{1 / 2}$, are listed. For each of the emission lines of the present study three rows are displayed: the fit parameters for a single Gaussian fit of the line profile (SG, top row) and two rows for the core component (CC, middle row) and the tail component (TC, bottom row) of the double Gaussian fits. The first column lists the radiances, $I$. In the second column the ratio of the tail component to the total line emission, $R$, is given, which applies only to the double Gaussian fit. The third column shows the relative shift, $v$, of the single Gaussian fit to the core component of the double Gaussian fit in the top row, and the shift of the tail component to the core component, both of the double Gaussian fit, in the bottom row. Positive values correspond to redshifts. In the fourth column the line widths, $w$, corrected for instrumental broadening are listed. Finally the fifth column gives the $\chi_{\mathrm{r}}^{2}$ values for the single Gaussian fit (top row) and the double Gaussian fit (bottom row). For each parameter the median value of the fits for the network profiles is given along with the error of the fit and the standard deviation of the fit, $\sigma$, e.g. " $197 \pm 1332$ " stands for a median value of 197, an error of the fit of 13 and a standard deviation of the values in the profiles fitted of 32. This format is used for all entries in the list, except for $\chi_{\mathrm{r}}^{2}$, where only median values and standard deviations are given. The standard deviations $\sigma$ basically represent "solar noise", i.e. variations from one location to the other.

\begin{tabular}{|c|c|c|c|c|c|c|c|c|c|c|c|c|c|c|}
\hline line & $\begin{array}{r}\log T_{\mathrm{e}} \\
{[\mathrm{K}]}\end{array}$ & $\begin{array}{l}v_{\mathrm{s}} \\
{\left[\mathrm{km} \mathrm{s}^{-1}\right.}\end{array}$ & & $\begin{array}{l}\text { radiance } I \\
{\left[\mu \mathrm{W} /\left(\mathrm{m}^{2} \mathrm{sr}\right.\right.}\end{array}$ & $\begin{array}{l}\sigma_{I} \\
\mathrm{r})]\end{array}$ & $\begin{array}{r}\text { ratio } R \\
I_{\mathrm{TC}} /\left(I_{\mathrm{CC}}+\right.\end{array}$ & $\begin{array}{l}l= \\
\left.-I_{\mathrm{TC}}\right)\end{array}$ & $\sigma_{R}$ & $\begin{array}{l}\text { shift vs. CC } \\
\quad v\left[\mathrm{~km} \mathrm{~s}^{-1}\right]\end{array}$ & $\sigma_{v}$ & $\begin{array}{l}\text { line width } w \\
{\left[\mathrm{~km} \mathrm{~s}^{-1}\right]}\end{array}$ & $\sigma_{w}$ & $\chi_{\mathrm{r}}^{2}$ & $\sigma_{\chi_{\mathrm{r}}^{2}}$ \\
\hline $\begin{array}{l}\text { Si II } \\
1533 \AA\end{array}$ & 4.60 & 3.4 & $\begin{array}{l}S G: \\
\text { CC: } \\
\text { TC: }\end{array}$ & $\begin{array}{r}197 \pm 13 \\
198 \pm 10 \\
-\end{array}$ & $\begin{array}{l}32 \\
33\end{array}$ & \} & - & & $\begin{array}{r}0.0 \pm 2.3 \\
-\end{array}$ & 0.1 & $\begin{array}{r}20.3 \pm 1.9 \\
20.3 \pm 1.5 \\
-\end{array}$ & $\begin{array}{l}1.5 \\
1.7\end{array}$ & 0.9 & \\
\hline $\begin{array}{l}\text { C II } \\
1335 \AA\end{array}$ & 4.67 & 5.7 & $\begin{array}{l}S G: \\
\text { CC: } \\
\text { TC: }\end{array}$ & $\begin{array}{r}1041 \pm 19 \\
994 \pm 38 \\
54 \pm 9\end{array}$ & $\begin{array}{r}140 \\
139 \\
15\end{array}$ & $5.2 \pm$ & 3.3 & 1.5 & $\begin{array}{l}0.3 \pm 0.3 \\
6.5 \pm 13.0\end{array}$ & $\begin{array}{l}0.3 \\
5.8\end{array}$ & $\begin{array}{l}24.1 \pm 0.2 \\
23.2 \pm 0.3 \\
43.9 \pm 9.0\end{array}$ & $\begin{array}{l}0.8 \\
1.0 \\
4.6\end{array}$ & $\begin{array}{l}2.8 \\
1.7\end{array}$ & $\begin{array}{l}0.4 \\
0.3\end{array}$ \\
\hline $\begin{array}{l}\text { C III } \\
977 \AA\end{array}$ & 4.78 & 6.4 & $\begin{array}{l}S G: \\
\text { CC: } \\
\text { TC: }\end{array}$ & $\begin{array}{r}1014 \pm 43 \\
841 \pm 78 \\
190 \pm 19\end{array}$ & $\begin{array}{r}170 \\
151 \\
49\end{array}$ & $18.5 \pm$ & 2.3 & 3.9 & $\begin{array}{l}-0.4 \pm 0.2 \\
-3.4 \pm 0.9\end{array}$ & $\begin{array}{l}0.3 \\
3.2\end{array}$ & $\begin{array}{l}35.0 \pm 0.1 \\
29.9 \pm 0.3 \\
63.7 \pm 1.8\end{array}$ & $\begin{array}{l}2.4 \\
2.0 \\
7.9\end{array}$ & $\left\{\begin{array}{r}11.4 \\
1.9\end{array}\right.$ & $\begin{array}{l}4.0 \\
0.5\end{array}$ \\
\hline $\begin{array}{l}\text { Si IV } \\
1403 \AA\end{array}$ & 4.81 & 4.3 & $\begin{array}{l}S G: \\
\text { CC: } \\
\text { TC: }\end{array}$ & $\begin{array}{r}202 \pm 4 \\
152 \pm 12 \\
26 \pm 4\end{array}$ & $\begin{array}{r}29 \\
38 \\
8\end{array}$ & $14.8 \pm$ & 7.4 & 3.7 & $\begin{array}{l}-1.0 \pm 1.4 \\
-7.1 \pm 5.3\end{array}$ & $\begin{array}{l}0.9 \\
6.4\end{array}$ & $\begin{array}{l}28.2 \pm 0.7 \\
25.1 \pm 2.2 \\
44.7 \pm 7.2\end{array}$ & $\begin{array}{l}1.0 \\
1.6 \\
6.7\end{array}$ & $\begin{array}{l}0.7 \\
0.7\end{array}$ & $\begin{array}{l}0.2 \\
0.1\end{array}$ \\
\hline $\begin{array}{l}\text { C IV } \\
1548 \AA\end{array}$ & 5.00 & 8.3 & $\begin{array}{l}S G: \\
\text { CC: } \\
\text { TC: }\end{array}$ & $\begin{array}{r}1001 \pm 68 \\
920 \pm 94 \\
350 \pm 43\end{array}$ & $\begin{array}{l}304 \\
261 \\
109\end{array}$ & $27.6 \pm$ & 4.3 & 11.6 & $\begin{array}{l}-0.3 \pm 0.4 \\
-1.1 \pm 1.1\end{array}$ & $\begin{array}{r}1.6 \\
10.0\end{array}$ & $\begin{array}{l}30.7 \pm 0.2 \\
25.2 \pm 0.3 \\
53.4 \pm 3.5\end{array}$ & $\begin{array}{l}4.3 \\
4.7 \\
8.7\end{array}$ & $\begin{array}{l}1.8 \\
1.1\end{array}$ & $\begin{array}{l}1.2 \\
0.6\end{array}$ \\
\hline $\begin{array}{l}\text { O IV } \\
790 \AA\end{array}$ & 5.27 & 9.8 & $\begin{array}{l}S G: \\
\text { CC: } \\
\text { TC: }\end{array}$ & $\begin{array}{r}259 \pm 5 \\
199 \pm 9 \\
49 \pm 5\end{array}$ & $\begin{array}{l}58 \\
52 \\
27\end{array}$ & $19.0 \pm$ & 6.6 & 6.7 & $\begin{array}{r}0.2 \pm 1.3 \\
-7.1 \pm 6.8\end{array}$ & $\begin{array}{l}1.5 \\
6.1\end{array}$ & $\begin{array}{l}39.3 \pm 0.8 \\
33.3 \pm 1.8 \\
79.2 \pm 9.6\end{array}$ & $\begin{array}{r}1.3 \\
2.2 \\
14.6\end{array}$ & $\begin{array}{l}1.4 \\
0.7\end{array}$ & $\begin{array}{l}0.6 \\
0.3\end{array}$ \\
\hline $\begin{array}{l}\text { O VI } \\
1032 \AA\end{array}$ & 5.45 & 12.0 & $\begin{array}{l}S G: \\
\text { CC: } \\
\text { TC: }\end{array}$ & $\begin{array}{r}389 \pm 10 \\
341 \pm 28 \\
55 \pm 7\end{array}$ & $\begin{array}{r}138 \\
123 \\
22\end{array}$ & $13.9 \pm$ & 5.4 & 3.3 & $\begin{array}{l}-0.3 \pm 0.4 \\
-3.0 \pm 2.5\end{array}$ & $\begin{array}{l}0.5 \\
4.6\end{array}$ & $\begin{array}{l}38.0 \pm 0.3 \\
34.3 \pm 0.7 \\
71.3 \pm 5.7\end{array}$ & $\begin{array}{r}3.4 \\
2.7 \\
17.7\end{array}$ & $\left\{\begin{array}{l}3.8 \\
1.2\end{array}\right.$ & $\begin{array}{l}2.9 \\
0.3\end{array}$ \\
\hline $\begin{array}{l}\text { Ne VIII } \\
770 \AA\end{array}$ & 5.81 & 16.4 & $\begin{array}{l}S G: \\
\text { CC: } \\
\text { TC: }\end{array}$ & $\begin{array}{r}120 \pm 4 \\
106 \pm 8 \\
15 \pm 9\end{array}$ & $\begin{array}{r}19 \\
17 \\
5\end{array}$ & \} $12.9 \pm$ & 18.6 & 3.2 & $\begin{array}{r}-0.8 \pm 6.3 \\
6.5 \pm 23.0\end{array}$ & $\begin{array}{r}5.8 \\
55.5\end{array}$ & $\begin{array}{l}33.6 \pm 1.3 \\
31.3 \pm 2.5 \\
33.3 \pm 23.5\end{array}$ & $\begin{array}{l}1.7 \\
2.4 \\
9.3\end{array}$ & $\left\{\begin{array}{l}1.1 \\
0.9\end{array}\right.$ & $\begin{array}{l}0.2 \\
0.3\end{array}$ \\
\hline
\end{tabular}

previous studies (Chae et al. 1998). In contrast, the non-thermal line width of the tail component shows a monotonic increase that is basically a power law of the temperature (thick curve in Fig. 7).

Doppler shift variation. The variation of the relative shift of the tail component to the core component is shown in Fig. 8. Negative values correspond to a tail component blueshifted with respect to the line core. The plot gives the impression that the tail components in the middle transition region are systematically blueshifted compared to the core.

\subsection{Results for Ne VIII}

For the Ne VIII coronal line a double Gaussian fit is not much more significant than a single Gaussian fit $\left(\chi_{\mathrm{r}}^{2}=0.9\right.$ compared to 1.1, cf. Table 1 ). The wide tail component is, if present at all, only very weak and not detectable in the data set discussed here. Instead of a wide component a second Gaussian is often fitted in the red wing of the line profile of comparable or smaller width than the line core component, producing a "bump" in the red wing (Fig. 3).

It might also be possible that in areas where the chromospheric network is bright but the coronal emission is 


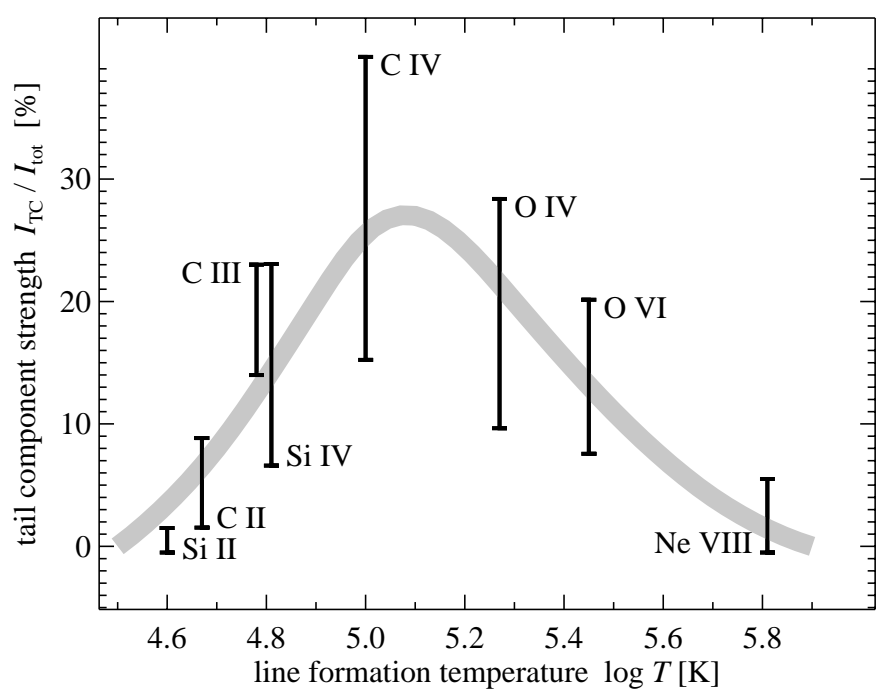

Fig. 6. Contribution of the broad spectral component, $I_{\mathrm{TC}}$, to the total emission in transition region lines, $I_{\mathrm{tot}}=I_{\mathrm{CC}}+I_{\mathrm{TC}}$, as a function of line formation temperature. Plotted is the mean ratio of the intensities in per cent for network locations in the data. The bars basically show "solar noise", see Sect. 2.5. The thick curve shows the estimated mean trend of the strength with temperature.

weak, the Ne VIII line, observed in second order here, is blended by five chromospheric lines of Si I seen in first order from $1540.3 \AA$ to $1541.6 \AA$. According to HRTS spectra these lines have roughly the same intensity, which is well below $10 \%$ of the Ne VIII line (Brekke 1993; Hassler et al. 1999, their Fig. 2b). These Si I lines are shifted with respect to the Ne VIII line at rest $(770.428 \AA$, Peter \& Judge 1999 ) by roughly $-100,-20,+20,+100$ and $+140 \mathrm{~km} \mathrm{~s}^{-1}$. Taking into account that NeVIII is blueshifted on the disk by about $3 \mathrm{kms}^{-1}$ (Peter 1999; Peter \& Judge 1999) it might be possible that the "bumps", typically at $5 \pm 20 \mathrm{~km} \mathrm{~s}^{-1}$ towards the red (cf. Table 1) are due to the Si I blends. However, the question remains why there is only one "bump" visible. If e.g. the very strong "bump" seen in the right example of Fig. 3 would be due to a Si I blend at $+20 \mathrm{~km} \mathrm{~s}^{-1}$, why there is no effect of comparable strength seen due to the Si I blends at $-100,+100$ and $+140 \mathrm{~km} \mathrm{~s}^{-1}$ ? In conclusion, more work needs to be done to explore the nature of these "bumps" in Ne VIII, especially whether these are simply a line blend or a physical effect in the solar corona.

Nevertheless, extrapolating the trend in line width towards coronal temperatures (thick dotted curve in Fig. 7) suggests a Ne VIII tail component non-thermal width of $\approx 100 \mathrm{~km} \mathrm{~s}^{-1}$. Assuming an upper limit of the strength of $5 \%$ leads to a peak intensity of the tail component below $1 \%$ of the one of the core component. As the peak intensity of Ne VIII is at most a factor of 100 stronger than the continuum near $770 \AA$ this implies that such a broad component is not detectable on the disk. Above the limb, though, a tail component contributing $20 \%$ to the total intensity can be seen in Ne VIII with a non-thermal broadening of 85 to $135 \mathrm{~km} \mathrm{~s}^{-1}$ (Wilhelm 1999), which is consistent with the

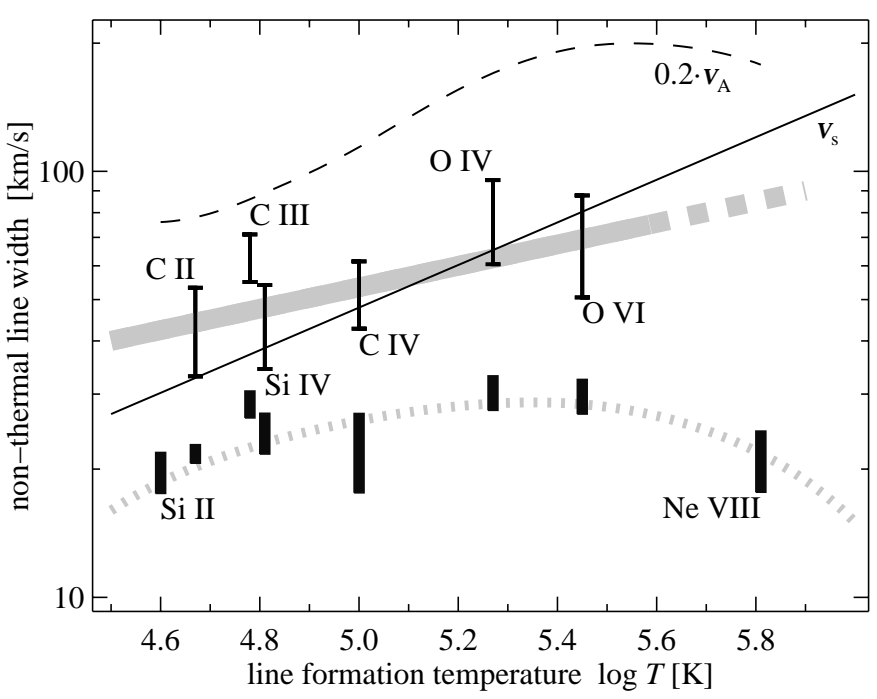

Fig. 7. Non-thermal line width of the cores of the line profiles (rectangles) and the broad components (bars) as a function of line formation temperature. As in Fig. 6 the rectangles and bars represent the "solar noise", see Sect. 2.5. The thick solid curve shows the trend of the width of the broad component with temperature $\left(\sim T^{1 / 4}\right)$, the extrapolation to coronal temperatures is shown as a thick dashed curve. The thick dotted curve shows a fit to the line core non-thermal widths as found in a previous study by Chae et al. (1998). The thin lines show the Alfvén speed $v_{\mathrm{A}}$ in a coronal funnel (dashed; reduced by a factor 5; following Hackenberg et al. 2000) and the adiabatic sound speed $v_{\mathrm{s}}$ (solid).

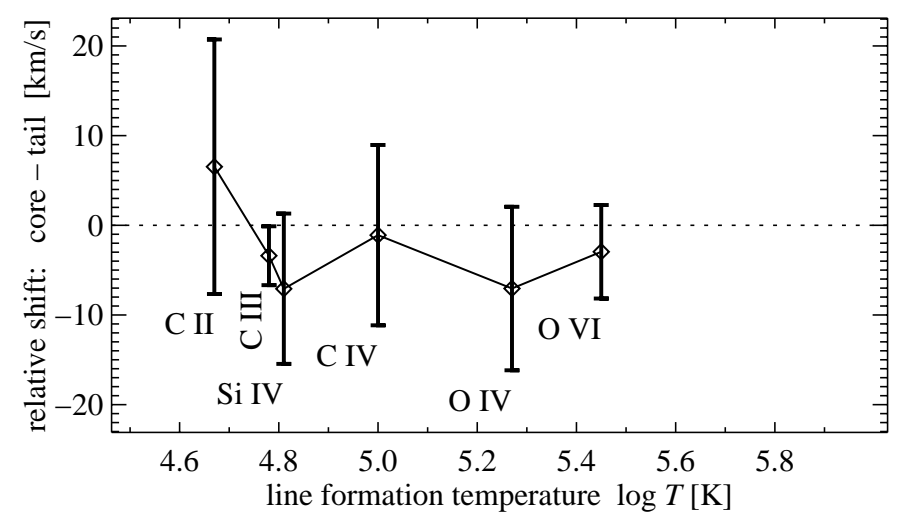

Fig. 8. Shift of the broad component to the narrow component. A negative value of the shift indicates that the broad component is blueshifted compared to the narrow component.

extrapolation shown in Fig. 7. If the line core were emitted from small loops and the tail component originates from the more extended funnels as proposed by Peter (2000), the contribution of the tail component would increase with altitude. This would then suggest that Ne VIII has a broad component also in on-disk spectra, even though it is not detectable because of the low line peak-to-continuum ratio. 


\section{Discussion}

The variation of strength and non-thermal width of the broad components with temperature provides a new challenge to model coronal heating. The power law of the nonthermal width of the tail components (Fig. 7) is an especially important feature to be understood theoretically.

The main question is what can be learned about the nature of the heating of the source region of the tail components. First one should note that the qualitatively different variation of the non-thermal width for the two spectral components indicates that they originate from physically different regions, as was hinted at in a previous study by Peter (2000). Additionally, in contrast to the line core the non-thermal velocity of the tail component is larger than the (adiabatic) sound speed in the lower transition region (Fig. 7, thin solid line).

It was suggested that (unresolved) super-sonic transients, e.g. explosive events, could produce the broad wings of CIV (Dere \& Mason 1993). Estimating the emission measures shows, however, that this is not the case, as long as a very large number of very small events do not exist (Peter 2000). Another candidate would be "blinkers" or other transition regions brightenings as observed e.g. by CDS/SOHO. A study of Harra et al. (2000) showed that in $\mathrm{OV}$ "network brightenings have energies an order of magnitude larger than the cell (inter-network) brightenings". That would nicely fit into the result of the present study that no (or only very few) tail components are found in the inter-network. However, Harra et al. (2000) observed only 0.02 such brightening events per CDS pixel $\left(4^{\prime \prime} \times 4^{\prime \prime}\right)$ during their nearly 2 hours study. Thus there is only 1 event every 100 hours in a $4^{\prime \prime} \times 4^{\prime \prime}$ region on the Sun. As the tail components are a common feature in the network, these events are to rare to explain them. The same applies for the EUV-blinkers.

\subsection{Waves passing coronal funnels}

If net motions like in explosive events do not cause the non-thermal broadening, one can look for magnetoacoustic waves (cf. the limits of a pure sound wave and an Alfvén wave, thin solid and dashed lines in Fig. 7). With the interpretation of the broad components originating from coronal funnels this would be consistent with the recent funnel model of Hackenberg et al. (2000) who suggest that the acceleration of the solar wind starting in the transition region is being driven by high-frequency Alfvén waves.

For the moment it should be assumed that the ions are at the same temperature as the electrons in the region of line formation. This seems to be justified as the lines form rather deep in the transition region, at most $10000 \mathrm{~km}$ above the photosphere. The densities should still be high enough to ensure a sufficient collisional coupling leading to thermal equilibrium between ions and electrons. This is in contrast to observations in the solar wind or the outer corona, but those are done at least one solar radius above

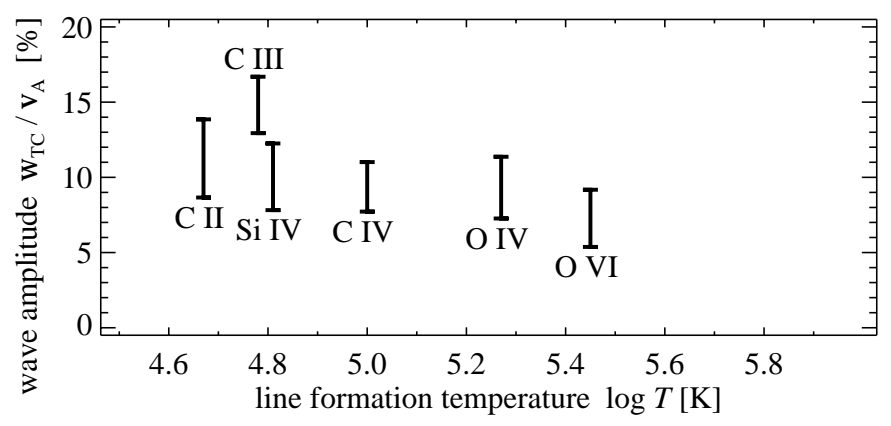

Fig. 9. Amplitude of an Alfén wave under the assumption that the non-thermal broadening of the tail component is caused by this wave (see Fig. 7; Alfvén speed from Hackenberg et al. 2000). See Sect. 4.1.

the limb at much lower densities. Therefore it is assumed that the lines have a thermal width corresponding to their formation temperature, and that the broadening in excess of this thermal width, leading to the observed line width, is due to any non-thermal effect, e.g. unresolved motions or waves. This is quickly illustrated e.g. for the tail component of CIV (cf. Table 1). The observed line width is $w=53.4 \mathrm{~km} \mathrm{~s}^{-1}$ and the ion sound speed $v_{\mathrm{s}}=\sqrt{k_{\mathrm{B}} T / m}$ is $8.3 \mathrm{~km} \mathrm{~s}^{-1}$. This results in a non-thermal width defined through $w^{2}=2 v_{\mathrm{s}}^{2}+\xi^{2}$ of $\xi=52.8 \mathrm{~km} \mathrm{~s}^{-1}$. Practically all of the line width is non-thermal broadening and only a tiny fraction is due to the temperature of the ions.

In the following it is assumed that all the non-thermal broadening is due to a passing wave. A wave with rootmean-square fluctuations $v_{\text {rms }}$ is causing a non-thermal broadening $\xi^{2}=2 / 3 v_{\mathrm{rms}}^{2}$. In an isobaric stratification like the transition region the density follows $\rho \sim 1 / T$ (ideal gas or adiabatic). For both a sound and an Alfvén wave the propagation speed varies as $v_{\text {prop }} \sim T^{1 / 2}$. Assuming a constant energy flux density $F \approx \rho v_{\text {rms }}^{2} v_{\text {prop }}$ of the wave (Brown \& Jordan 1981), the non-thermal broadening follows $\xi \sim T^{1 / 4}$. In conclusion, the observed variation of the non-thermal broadening of the broad components agrees well with a sound or an Alfvén wave with constant energy flux density crossing the transition region (Fig. 7, thick solid curve). As acoustic waves of an amplitude that large, i.e. larger than the sound speed, would be highly non-linear and damped very efficiently, an Alfvén wave should be favoured. For an Alfvén wave the energy flux density would be about $F_{\mathrm{A}} \approx 1000 \mathrm{~W} / \mathrm{m}^{2} B[\mathrm{G}]$, with the magnetic field $B$ measured in Gauss. This would be more than sufficient to heat the corona, even when considering the expansion of the funnels.

An Alfvén wave leading to a non-thermal broadening of the order of $50-100 \mathrm{~km} \mathrm{~s}^{-1}$, as it is the case for the tail components, would be highly non-linear. To calculate the wave amplitude, the Alfvén speed $v_{\mathrm{A}}$ from the coronal funnel model of Hackenberg et al. (2000) is used, which is over-plotted as a function of temperature in Fig. 7 (dashed line, $v_{\mathrm{A}}$ reduced by a factor of 5 ). The wave amplitude is the quotient of the non-thermal broadening, assumed to be due to the wave, and the Alfvén speed. As it can be seen 


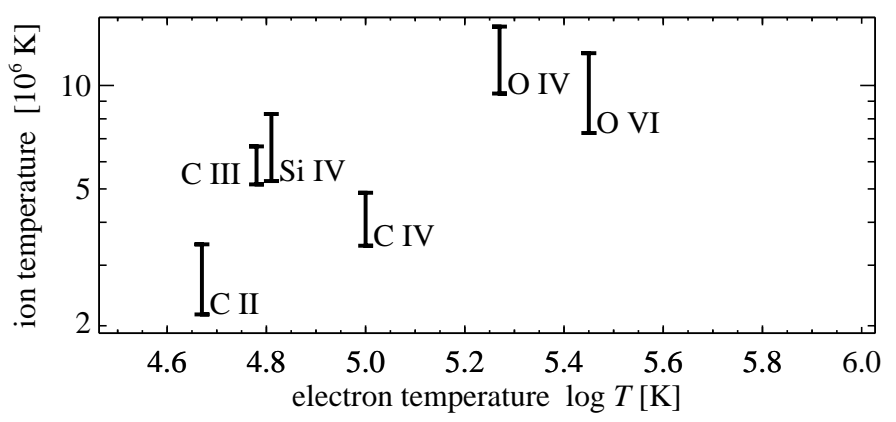

Fig. 10. Kinetic ion temperatures, if the tail component line widths as plotted in Fig. 7 are interpreted as due to thermal motions of the ions alone. In this case the ions would be much hotter than the electrons, just as it is observed in the upper corona and solar wind. See Sect. 4.2.

from Fig. 9, the wave amplitude is about 10\%, indicating the non-linear character of the wave, and the amplitude is slightly decreasing with temperature.

\subsection{Preferential ion heating in coronal funnels}

The above discussion is built on the assumption that the ions have the same temperature as the electrons in the line formation region, and that the non-thermal broadening of the tail components is due to a passing wave. However, one might think also about e.g. Landau-damping of magnetoacoustic waves leading directly to heating of the ions and by this to temperatures of the ions in excess of the electrons. In that case the ions would be much hotter than the electrons already deep in the transition region. This might be possible in lower density regions, like in the (locally) open coronal funnels, where the collisional coupling might be too inefficient to keep the electrons and the minor species at the same temperature.

This is a well known effect in the solar wind, where the ion temperatures are roughly mass-proportional, and also in the upper corona, where remote sensing observations of UVCS/SOHO show temperatures of $\mathrm{O}^{5+}$ ions of the order of $4 \times 10^{8} \mathrm{~K}$ at about $1.5 R_{\odot}$ above the limb (e.g. Kohl et al. 1999), while the electron temperatures are below $10^{6} \mathrm{~K}$ (Wilhelm et al. 1998). Recently Vocks \& Marsch (2001) studied a semi-kinetic corona model including the transition region. They found that wave-ion interactions indeed lead to preferential ion heating deep in the transition region, and thus support the above interpretation of the broad tail components as ions having temperatures in excess of the electrons.

Interpreting the line widths of the second components (Table 1, Fig. 7) as a kinetic ion temperature leads to ion temperatures of a couple of $10^{6} \mathrm{~K}$ already deep in the coronal funnels, where the electron temperature (determining the line formation temperature ${ }^{1}$ ) is still well be-

\footnotetext{
${ }^{1}$ The line formation temperature is basically given by the ionisation equilibrium, with the ionisation being dominated by electron collisions. As the collisions depend on the relative speed, and the thermal speed of the electrons largely exceeds
}

low $5 \times 10^{5} \mathrm{~K}$ ! The resulting kinetic ion temperatures are plotted vs. the electron temperatures in Fig. 10.

Thinking of heating mechanisms like the highfrequency Alfvén wave heating proposed by Tu \& Marsch (1997) and Marsch \& Tu (1997), this would be no surprise. This result would have major impact on our understanding of the heating process for the minor ions in the solar corona, but also on our understanding of the heating of the corona and the acceleration of the solar wind in general.

To investigate this further more theoretical work is needed that is well beyond the scope of this paper.

\subsection{Intensity distribution}

Another interesting question concerns the variation of the strength of the broad components with temperature. From the chromosphere to the middle transition region the waves could steepen into shocks due to decreasing density, leading to a more effective emission and finally to a larger contribution of the broad components. The decreasing strength towards the corona does not have to imply that the source region of the broad components, the funnels, does not reach to temperatures that high. As the source regions of the two components are heated differently, the energy balance can be expected to be different, finally resulting in different density and pressure stratifications in magnetically (locally) open and closed structures (Holzer et al. 1997). Therefore the decreasing strength of the broad component in the upper transition region might simply be due to different emissivities in the source regions of the line core and the tail component.

\subsection{Coronal funnel Doppler shifts}

Except for $\mathrm{C}$ II from the lowermost transition region, the broad tail components are blueshifted compared to the core component (Fig. 8). Still, their net line shift is towards the red, as the line cores show redshifts of up to $15 \mathrm{~km} \mathrm{~s}^{-1}$ in the middle transition region (e.g. Peter \& Judge 1999). One has also to note that the uncertainties for the line shift are quite large (compared to the core component), because it is difficult to determine the position of a faint wide Gaussian below a narrow Gaussian.

This result does not show that all coronal funnels are connected to the solar wind and by this show a blueshift as a signature of an outflow. This is not expected, as the region studied in the present paper is quiet Sun at midlatitudes.

Nevertheless, there is a tendency of the tail components to be blueshifted relative to the core. This can be understood if the fraction of area showing blueshifts is larger for the coronal funnels than for the closed regions emitting the line cores. This might be an indication that at least some of the funnels in the observed region are

the ion thermal speed because of the low electron mass, the ionisation is basically set by the electron temperature and is largely independent of the temperature of the ions. 


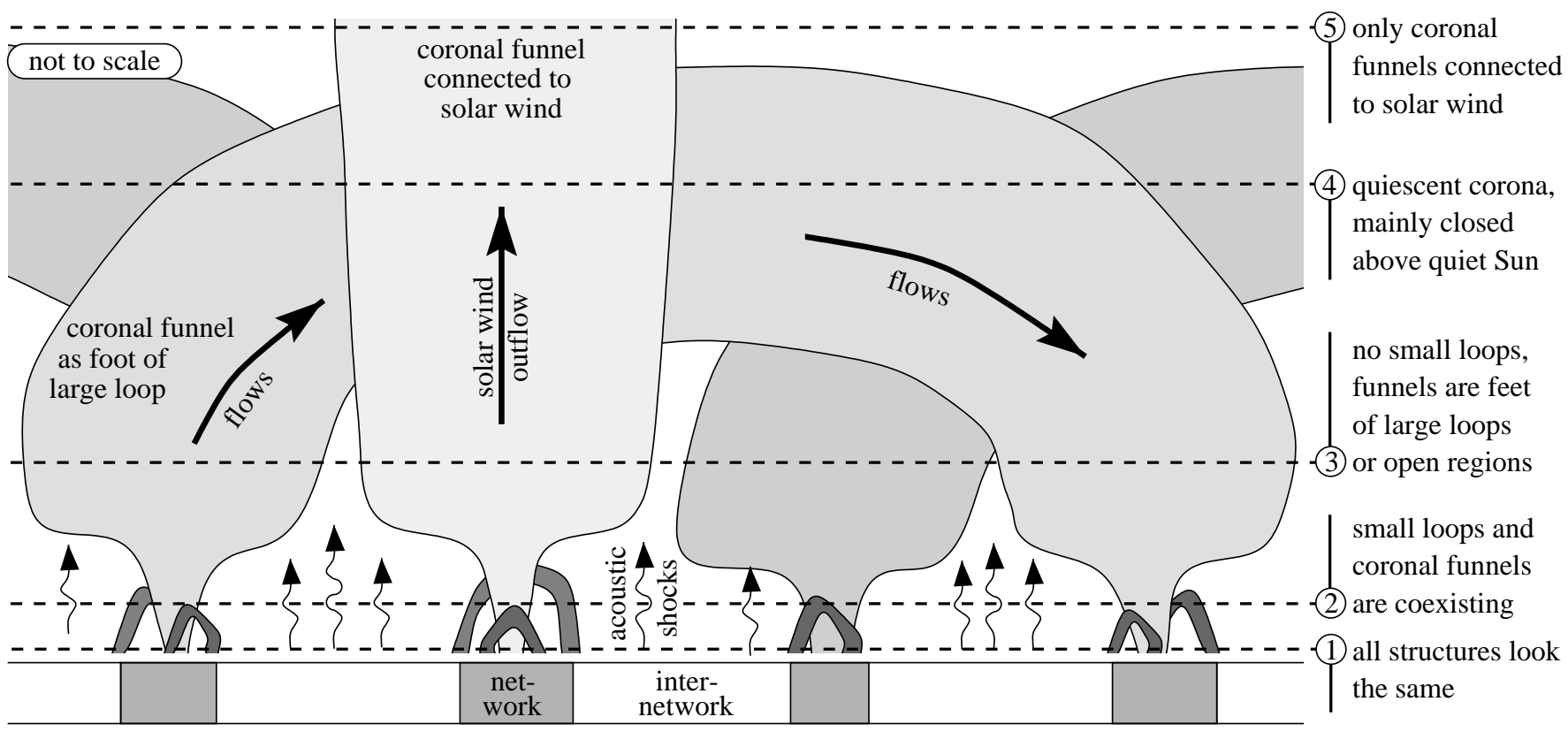

Fig. 11. Sketch of the structure of the transition region. This scenario and the labeling to the right is discussed in Sect. 4.5 .

connected to the solar wind, even though they are within the quiet Sun. But it might also indicate, that we see flows along large loops in the funnel-type feet of these loops. Because the loops are large, their feet can be distinguished and regions with a net upflow are found frequently. This interpretation is also supported by the previous study of Peter (2000).

More work is needed, especially on comparable data sets from coronal holes, to clarify this point.

\subsection{Transition region structure}

The structure of the transition region as suggested by the presented observations is sketched in Fig. 11 (the following numbers refer to the the levels indicated by the dashed lines in the figure).

(1) In the middle chromosphere up to approximately some $1000 \mathrm{~km}$ above the $\tau_{500 \mathrm{~nm}}=1$ level the feet of the various coronal magnetic structures in the network still look the same. The feet of the coronal funnels and the small coronal loops might have comparable size and undergo the same physical processes. The main distinction to be made in the chromosphere is between the magnetically dominated network and the inter-network dominated by the acoustic upward-propagating shocks as proposed by e.g. Carlsson \& Stein (1997).

(2) At higher altitudes, where the coronal funnels are starting to expand, the funnels are still "coexisting" with the smaller network loops. A possible scenario for the funnel expansion is based on the (horizontal) balance of the magnetic pressure due to the smaller network loops, which might reach some $5000 \mathrm{~km}$ in altitude, and on the vanishing power of the acoustic shocks in the non-magnetic inter- network. This also puts a rough estimate of the height of the funnel expansion to about $5000 \mathrm{~km}$.

(3) Well above, say $10000 \mathrm{~km}$, the funnels finally fill the whole volume of the corona. At this level all the funnels look the same, no matter whether they are open magnetic structures connected to the heliosphere or whether they are the base of large coronal loops spanning across one or more network elements.

(4) The funnels connected to the solar wind and those being the feet of large coronal loops are most probably heated by the same processes. This is suggested by the simple consideration that the chromosphere would not care much whether a magnetically closed or open structure is sitting above it, as the chromosphere is energetically and dynamically independent from the corona. In the open funnels the heating is used to power the solar wind. In large coronal loops the (slightly) different heating in the funnels sitting at the respective loop's feet in different network elements will lead to an asymmetry which will in turn power mass motions. These are frequently observed in large loops (e.g Brekke et al. 1997). Models for asymmetric heating are well studied e.g. by McClymont \& Craig (1987) or Klimchuk \& Mariska (1988).

(5) Above the quiet Sun, i.e. outside coronal holes and active regions, the large coronal loops are dominating and only very few funnels might be really magnetically open. However, considering the three-dimensional structure of the corona one should not rule out, that some of these open structures might exist even above the "quiet closed" Sun. Finally above the large coronal loops above the quiet Sun the open funnels from different regions will expand and merge to form the more or less uniform magnetic field of the Sun above some solar radii. 
This scenario should be considered only as a rough sketch. Many solar features are not specifically taken into account here. EUV-spicules (that might actually differ from the "traditional" spicules seen in $\mathrm{H} \alpha$ ) might especially play an important role (e.g. Wilhelm 2000). However, one might easily fit them into the network structures as yet another component.

\subsection{Relation to coronal hole blueshifts in Ne VIII}

In a study of Doppler shifts of the line core component of Ne VIII in a coronal hole and on the quiet Sun Hassler et al. (1999) found the strongest blueshifts in Ne VIII along network lanes defined by chromospheric emission (which is quite different from the Ne VIII emission pattern). One has to note that Ne VIII is also blueshifted on the quiet Sun in closed regions (Peter 1999; Peter \& Judge 1999). Their conclusion was that the solar wind outflow is concentrated along these chromospheric network lanes, especially where lanes come together.

Wilhelm et al. (2000) proved that the strongest blueshifts, interpreted as the highest outflow velocities, appear in those regions where the Sun is darkest in Ne viII. This nicely fits into the already mentioned results of Holzer et al. (1997), namely that open regions, where the solar wind is accelerated, should be a factor of roughly 10 fainter that closed regions - in open regions $90 \%$ of the energy is used to power the solar wind (Hansteen \& Leer 1995), and therefore only a fraction of the energy is left for line emission.

Unfortunately, by using existing data it is not possible to definitely conclude if there is a tail component present for Ne VIII and if so, what its width might be, even though there is evidence for its presence (see Sect. 3.3). Nevertheless, it seems plausible, that in coronal holes a weak tail component from funnels is also present. Due to the open field geometry in the holes, more small scale features might be connected to the solar wind and therefore show blueshifted Ne VIII emission with a relatively narrow line profile. In this sense the results presented in the present paper fit into the previous ones of Hassler et al. (1999) and Wilhelm et al. (2000) and are complementary to them.

\subsection{Stellar transition regions}

As yet, stellar work on the broad spectral components has concentrated on the analysis of C IV and Si IV lines. The present discussion has shown that it is of importance to also investigate other lines formed throughout the whole transition region, from $40000 \mathrm{~K}$ to a million degrees. Hopefully this work will encourage new stellar EUV observations. It would be of great interest to investigate the possibility that there is also a power law for the variation of the tail component non-thermal width with temperature for other stars, and if so, how the slope of the power law changes with, e.g., stellar activity. This would provide additional input to come closer to a solution of the coronal heating problem.

\section{Conclusions}

The SUMER data presented above show for the first time that the presence of a broad second spectral component is a general feature of emission lines originating from the chromosphere - corona transition region above the magnetically dominated chromospheric network. A power law of $T^{1 / 4}$ was found for the non-thermal width of the tail component, indicating that the broadening of the tail component is due to a passing magneto-acoustic wave. The results are consistent with the interpretation that the tail components originate from coronal funnels heated by dissipation of Alfvén waves. One should also consider the possibility that the tail component line width is a consequence of high ion temperatures of the order of some million degrees already deep in the transition region, where the electron temperature is still below half a million degrees. After the first report on broad spectral components in the transition region about two decades ago, the observational basis is now finally available to build new multicomponent models of the solar transition region.

Acknowledgements. Special thanks are due to Reiner Hammer, Eckart Marsch and Scott McIntosh for very valuable discussions and to Klaus Wilhelm and Reiner Hammer for reading the manuscript. Sincere thanks are due to an anonymous referee, in particular for the suggestion to extend the presentation of the data. I would like to thank the SUMER team at MPAe in Lindau/Germany for their good support on technical problems and especially the PI Klaus Wilhelm for the open data policy. SUMER is financially supported by DLR, CNES, NASA and the ESA PRODEX programme (Swiss contribution).

\section{References}

Bevington, P. R., \& Robinson, D. K. 1992, Data Reduction and Error Analysis for the Physical Sciences (McGraw-Hill, New York)

Brekke, P. 1993, ApJS, 87, 443

Brekke, P., Kjeldseth-Moe, O., \& Harrison, R. A. 1997, Sol. Phys., 175, 511

Brown, A., \& Jordan, C. 1981, MNRAS, 196, 757

Carlsson, M., \& Stein, R. F. 1997, ApJ, 481, 500

Chae, J., Schühle, U., \& Lemaire, P. 1998, ApJ, 505, 957

Charbonneau, P. 1995, ApJS, 101, 309

Dere, K. P., Bartoe, J.-D. F., \& Brueckner, G. E. 1989, Sol. Phys., 123, 41

Dere, K. P., \& Mason, H. E. 1993, Sol. Phys., 144, 217

Dowdy, J. F., Rabin, D., \& Moore, R. L. 1986, Sol. Phys., 105, 35

Gabriel, A. H. 1976, Phil. Trans. Roy. Soc. Lond., A 281, 339

Hackenberg, P., Marsch, E., \& Mann, G. 2000, A\&A, 360, 1139

Hansteen, V. H., \& Leer, E. 1995, J. Geophys. Res., 100, 21577

Harra, L. K., Gallagher, P. T., \& Phillips, K. J. H. 2000, A\&A, 362,371

Hassler, D. M., Dammasch, I. E., Lemaire, P., et al. 1999, Science, 283, 810 
Holzer, T. E., Hansteen, V. H., \& Leer, E. 1997, in Cosmic winds and the heliosphere, ed. J. R. Jokipii, C. P. Sonett, \& M. S. Giampapa (Univ. of Ariziona Press, Tucson), 239 Innes, D. E., Inhester, B., Axford, W. I., \& Wilhelm, K. 1997, Nature, 386, 811

Kjeldseth Moe, O., \& Nicolas, K. R. 1977, ApJ, 211, 579

Klimchuk, J. A., \& Mariska, J. T. 1988, ApJ, 328, 334

Kohl, J. L., et al. 1999, ApJ, 510, L59

Marsch, E., \& Tu, C.-Y. 1997, A\&A, 319, L17

McClymont, A. N., \& Craig, I. J. D. 1987, ApJ, 312, 402

Peter, H. 1999, ApJ, 516, 490

Peter, H. 2000, A\&A, 360, 761

Peter, H., \& Judge, P. G. 1999, ApJ, 522, 1148

Porter, J. G., Moore, R. L., Reichmann, E. J., et al. 1987, ApJ, 323,380
Priest, E. R., et al. 1998, Nature, 393, 545

Reeves, E. M. 1976, Sol. Phys., 46, 53

Tu, C.-Y., \& Marsch, E. 1997, Sol. Phys., 171, 363

Vocks, C., \& Marsch, E. 2001, Geophys. Res. Lett., in press

Wilhelm, K. 1999, Astrophys. Space Sci., 264, 43

Wilhelm, K. 2000, A\&A, 360, 351

Wilhelm, K., Curdt, W., Marsch, E., et al. 1995, Sol. Phys., 162,189

Wilhelm, K., Dammasch, I. E., Marsch, E., \& Hassler, D. M. 2000, A\&A, 353, 749

Wilhelm, K., Marsch, E., Dwivedi, B. N., et al. 1998, ApJ, 500, 1023

Wood, B. E., Linsky, J. L., \& Ayres, T. R. 1997, ApJ, 478, 745 